\title{
Conceptual design of the CBM-TOF wall with real- size high-rate MRPC modules based on the newly developed Chinese doped glass
}

\author{
Jingbo Wang ${ }^{1}$ \\ Department of Engineering Physics, Tsinghua University, Key Laboratory of Particle \& Radiation \\ Imaging, Ministry of Education \\ Beijing 100084, China \\ E-mail:wjb04@mails.tsinghua.edu.cn
}

\author{
Yi Wang ${ }^{a}$, Huangshan Chen ${ }^{a}$, Xingming Fan ${ }^{a}$, D.Gonzalez-Diaz ${ }^{\mathrm{a}, b, c}$, Yuanjing Li ${ }^{\mathrm{a}}$, \\ Jianping Cheng ${ }^{\text {a }}$ \\ ${ }^{a}$ Department of Engineering Physics, Tsinghua University, Key Laboratory of Particle \& Radiation \\ Imaging, Ministry of Education \\ Beijing 100084, China \\ ${ }^{b}$ GSI Helmholtz Center for Heavy Ion Research, Darmstadt, Germany \\ ${ }^{c}$ Laboratorio de Física Nuclear y Altas Energías, Universidad de Zaragoza, Zaragoza, Spain
}

\begin{abstract}
The Compressed Baryonic Matter experiment at the future Facility for Antiproton and Ion Research will use a time-of-flight (TOF) wall for hadron identification, based on the MRPC technology. The wall will be placed at $10 \mathrm{~m}$ distance from the target covering an area on the order of $150 \mathrm{~m}^{2}$. We propose here a realistic design for building the TOF wall by resorting to a single technology based on low-resistivity doped glass and relying on small structural modifications of the modules developed and tested during the last two years. The performance of real-size modules is also summarized.
\end{abstract}

XI workshop on Resistive Plate Chambers and Related Detectors (RPC2012)

INFN-Laboratori Nazionali di Frascati, Italy

February 5-10, 2012

speaker 


\section{Introduction}

The Compressed Baryonic Matter (CBM) experiment at the future Facility for Antiproton and Ion Research (FAIR) in Darmstadt, Germany, aims at exploring the QCD phase diagram under extremely high net baryon densities and moderate temperatures [1]. For the purpose of hadron identification, a Time-of-Flight (TOF) wall placed $10 \mathrm{~m}$ away from the target and with a polar angle coverage of $2.5^{\circ}-25^{\circ}$, is imperative. The wall features a TOF resolution better than $80 \mathrm{ps}$ and an occupancy per cell below $5 \%$ over an area of $150 \mathrm{~m}^{2}$. The challenge is to keep high efficiencies (above 90\%) and good time resolutions (less than 80ps) at particle fluxes up to $20 \mathrm{kHz} / \mathrm{cm}^{2}$, which is not accessible to conventional float-glass MRPCs. For this purpose, the R\&D activities focus on the development of high-rate MRPCs [2-6]. In order to improve the rate capability, a type of low-resistivity doped glass with bulk resistivity on the order of $10^{10}$ $\Omega \mathrm{cm}$ was produced at Tsinghua University [4]. Two real-size high rate prototypes based on this material were developed and tested in-beam.

The current conceptual design of the CBM-TOF wall foresees two extreme regions: an outermost region (low rate/ low multiplicity) covered by float glass RPCs in multi-strip fashion, and a central region (high rate/high multiplicity) consisting of densely packed read-out cells made of low resistivity electrodes [7]. In this contribution we propose a slightly different conceptual design for building the TOF wall by resorting to a single technology that is based on the newly developed low-resistivity doped glass. The design strongly relies on the two modules developed and tested during the last two years, upon minor structural modifications in some regions.

For completeness, we summarize our latest results concerning the rate capability of two real-size modules aimed at the coverage of the aforementioned, very distinct, regions. The latest measurements were performed with a continuous electron beam from the Electron Linac with high Brilliance and low Emittance (ELBE) facility at the Helmholtz-Zentrum DresdenRossendorf (HZDR), in April 2011. They essentially confirm our earlier results as obtained for hadrons under uniform irradiation. A more comprehensive discussion of these results is currently under preparation.

\section{A Conceptual design of the CBM TOF wall}

In the current conceptual design, the CBM TOF wall consists of an array of 80 supermodules $(\mathrm{SM})$ with an active area of $1.5 \mathrm{~m} \times 1 \mathrm{~m}$ each. These SMs are mechanically arranged along nine columns and form four "rate regions", as shown in Fig. 1 [7]. In rate regions 1-3, the particle flux ranges from $3.5 \mathrm{kHz} / \mathrm{cm}^{2}$ to $25 \mathrm{kHz} / \mathrm{cm}^{2}$, which is not achievable by MRPCs made of conventional float glass; thus, high-rate MRPCs with low-resistivity glass electrode are presently the best choice. In rate region 4 the average particle flux is about $1 \mathrm{kHz} / \mathrm{cm}^{2}$, so MRPCs made out of float glass might be used. Perhaps a moderate detector warming $\left(\sim 10^{\circ} \mathrm{C}\right)$ will be then needed in order to comfortably secure such a rate capability. 


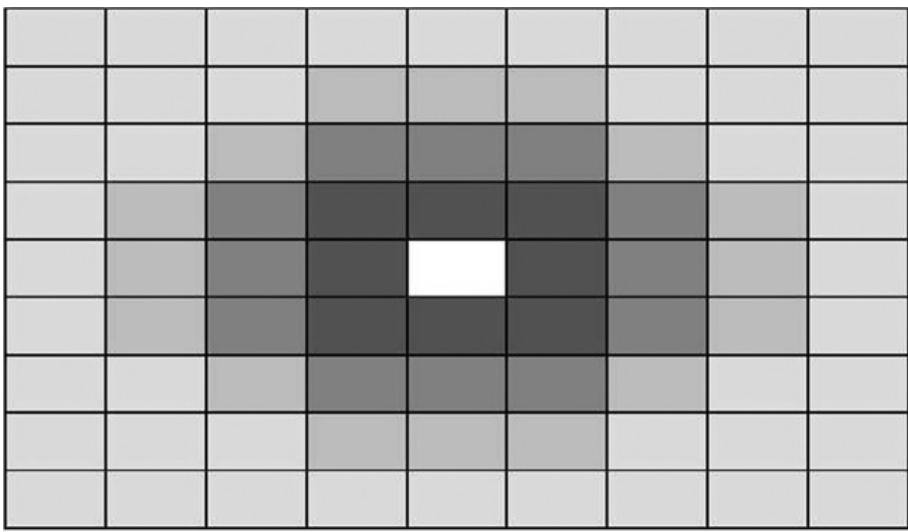

rate region 1: $8.0 \mathrm{kHz} / \mathrm{cm}^{2}-25 \mathrm{kHz} / \mathrm{cm}^{2}$, \#SM:8 rate region 2: $3.5 \mathrm{kHz} / \mathrm{cm}^{2}-8.0 \mathrm{kHz} / \mathrm{cm}^{2}$, \#SM:12 rate region $3: 1.5 \mathrm{kHz} / \mathrm{cm}^{2}-3.5 \mathrm{kHz} / \mathrm{cm}^{2}$, \#SM:16 rate region $4: 0.5 \mathrm{kHz} / \mathrm{cm}^{2}-1.5 \mathrm{kHz} / \mathrm{cm}^{2}$, \#SM:44

Fig. 1 Particle load on the 80 super-modules, separated in four regions with similar flux. (Figure from [7]).

We propose here a different conceptual design for building the TOF wall by resorting to a single technology based on low-resistivity doped glass and relying on small structural modifications of the two modules developed and tested during the last two years. Compared to ordinary float glass, however, the technology of production of this new glass is less mature, so it is still difficult to produce glass plates larger in size than $50 \mathrm{~cm} \times 50 \mathrm{~cm}$. For this reason, we propose to build the TOF wall with MRPCs not exceeding those dimensions. In this contribution we present a realistic detector layout for the CBM-TOF wall based on five different types of fundamental building blocks, or modules, as shown in Fig.2.

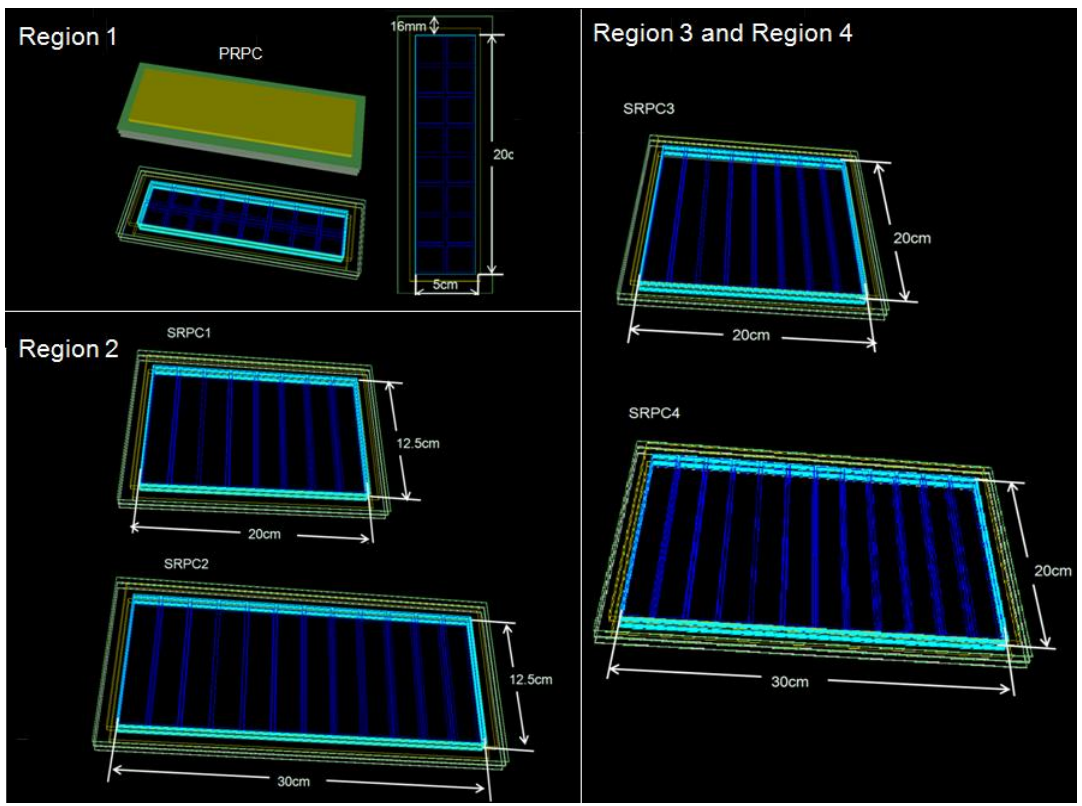

Fig. 2 Five different types of fundamental MRPC modules: the pad counters (PRPCs) will be used in "rate region" 1 to cover the highest particle flux up to $25 \mathrm{kHz} / \mathrm{cm}^{2}$ and the strip counters (SRPCs) will be used in the remaining 3 regions under a particle flux from $0.5-8 \mathrm{kHz} / \mathrm{cm}^{2}$. 


\subsection{Conceptual design of the Super Modules (SM)}

Region 1 will be covered by pad-readout modules. Based on real-size counters tested both with proton and electron beams, a 16-pad module (PRPC) is foreseen for constructing each Super-Module (SM). The geometrical layout of the module is illustrated in Fig. 2. This counter is arranged in a double stack configuration with a gas gap of $0.22 \mathrm{~mm}$ ensured by nylon monofilaments. It has an active area of $20 \mathrm{~cm} \times 5 \mathrm{~cm}$ and it is sub-divided in sixteen $2.2 \mathrm{~cm} \times$ $2.2 \mathrm{~cm}$ pads with an interval between pads of $3 \mathrm{~mm}$. The assembly design of the $\mathrm{SM}$ is depicted in Fig. 3. The SM consists of 150 MRPC modules and 2400 pads and the total active area is $1.5 \mathrm{~m}^{2}$. Region 1 can be assembled with $8 \mathrm{SMs}$ and the total number of electronic channels is 19200.

For region 2, high-rate strip counters with two characteristic sizes are used (SRPC1 and SRPC2). The counters have a gap width of $0.25 \mathrm{~mm}$. Fig. 3 shows the assembly design of the SM for region 2: 8 large counters (SRPC2) for constructing the middle column and 48 small counters (SRPC1) for the remaining columns are needed. Region 2 can be assembled with 12 SMs and the total number of electronic channels is 11520.

Region 3 and region 4 can share identical geometrical structure. The basic structure of counters here is the same as that designed for Region 2 except for a slightly larger size. For one SM, 5 big counters (SRPC4) are needed in order to build the middle column and 30 small counters (SRPC3) are needed for the remaining columns, as shown in Fig. 3. Region 3 and region 4 both need 60 SMs in total and the sum of the electronic channels is 36000 .

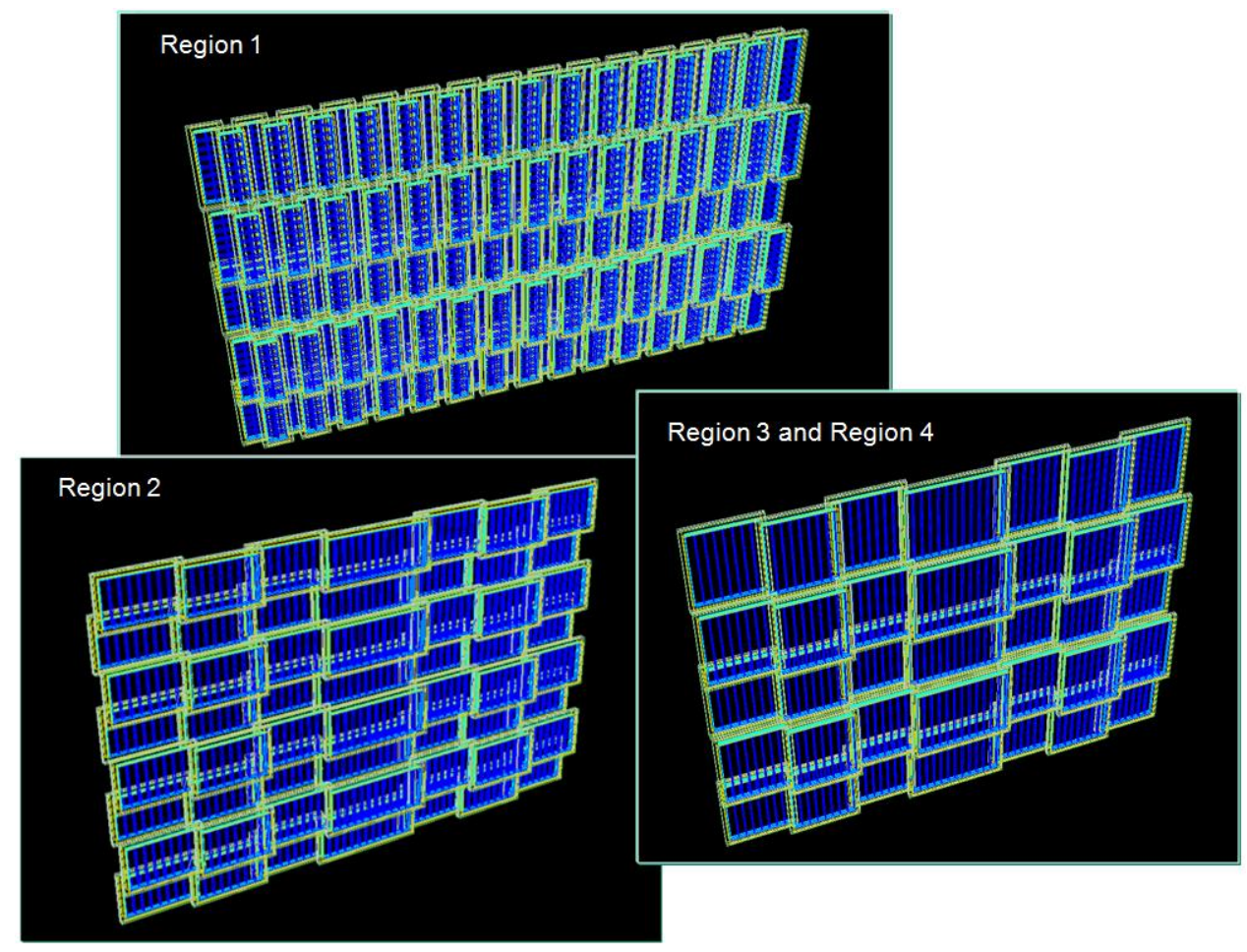

Fig. 3 Conceptual design of the Super-Modules for the 4 rate regions. 
The number of channels of this proposal is fully compatible with the geometry presently used for MC simulations [9] (65000) and within 10\% of the latest geometry given in [7]. Such a granularity ensures a 3-5\% occupancy for the harshest scenario stemming from Au+Au central collisions at the highest SIS-300 energy. The specifications of this realistic, yet largely conceptual, design are summarized in Table. 1.

\begin{tabular}{|l|l|l|l|l|}
\hline & Region 1 & Region 2 & Region 3 and 4 & TOF wall \\
\hline $\begin{array}{l}\text { Dimension of glass } \\
\text { plate }[\mathrm{mm}]\end{array}$ & $\begin{array}{l}212 \times 62 \times 1 \\
206 \times 56 \times 0.7\end{array}$ & $\begin{array}{l}212 \times 137 \times 1 \\
312 \times 137 \times 1 \\
206 \times 131 \times 0.7 \\
306 \times 131 \times 0.7\end{array}$ & $\begin{array}{l}212 \times 212 \times 1 \\
312 \times 212 \times 1 \\
206 \times 206 \times 0.7 \\
306 \times 206 \times 0.7\end{array}$ & $\begin{array}{l}\text { Maximum } \\
\text { dimension: } \\
312 \times 212 \times 1\end{array}$ \\
\hline Area of glass [m $\left.{ }^{2}\right]$ & 174 & 239 & 1166 & Sum: 1579 \\
\hline $\begin{array}{l}\text { Weight of SM } \\
\text { including box }[\mathrm{kg}]\end{array}$ & 125 & 131 & 129 & $\begin{array}{l}\text { Maximum } \\
\text { weight: } 131\end{array}$ \\
\hline $\begin{array}{l}\text { Total electronic } \\
\text { channels }\end{array}$ & 19200 & 11520 & 36000 & Sum: 66720 \\
\hline
\end{tabular}

Table. 1 Specifications of the realistic conceptual design proposed for the CBM TOF wall.

\section{Beam test @ ELBE}

In April 2009, a small 10-gap module was tested at GSI-Darmstadt with a pulsed proton beam of $2.5 \mathrm{GeV}$ and the successful results published in [4]. Due to the small size of the counter, and the fact that measurements were done off-axis, the irradiation can be considered to be largely uniform over its surface. By defining the rate capability as the particle flux at which a resolution deterioration by 20 ps or a $5 \%$ efficiency drop is observed, a rate capability of 25 $\mathrm{kHz} / \mathrm{cm}^{2}$ can be estimated.

Recently, our effort is to realistically adapt the MRPC geometry to suit the conceptual design of the TOF wall, as of [7], along the lines given in previous section. Two real-size counters made of low-resistivity doped glass were developed at Tsinghua University and the electron beam of the Electron Linac with high Brilliance and low Emittance (ELBE) facility [8] at Helmholtz-Zentrum Dresden-Rossendorf (HZDR) was used in April 2011 to test the performance. The pad-readout counter (PRPC\#1), is almost identical to the one illustrated in Fig. 2 but with two pads less ( $2 \times 6$ pads). The other real-size counter, based on the multi-strip concept, is structurally identical to the multi-strip modules shown in Fig.2 but with a smaller number of strips (3).

\subsection{Test setup}

The tests were performed with a low-intensity electron beam of $30 \mathrm{MeV}$ kinetic energy. The radio frequency signal (RF) of the electron source was used as the reference time, featuring an excellent resolution of about 35ps. The experimental setup is shown in Fig. 4. The MRPC and scintillator S6 were both placed on a moving platform, thus the counter could be moved vertically and horizontally via remote control. The trigger was determined by the coincidence between all the scintillators. A thin scintillator was moved horizontally and vertically to scan the beam profile and a $\sigma \approx 1.1 \mathrm{~cm}$ (hor) $\times 1.5 \mathrm{~cm}$ (vert) physical beam size was thus estimated, RPC 
upstream. However, a broader horizontal profile was determined by the RPC itself (self-trigger) from the time difference of both strips ends, $(\sigma=2.1 \mathrm{~cm})$. Since the position resolution of this counter+electronics is known to be $0.8 \mathrm{~cm}$, we attribute the effect to multiple scattering, that is expected to be much larger here as compared to the hadron case[4], due to the very low momentum $(p=30 \mathrm{MeV} / \mathrm{c}){ }^{2}$ The observed effect is indeed compatible with the one expected from the setup geometry and the material budget of the RPC itself and the first scintillator upstream.

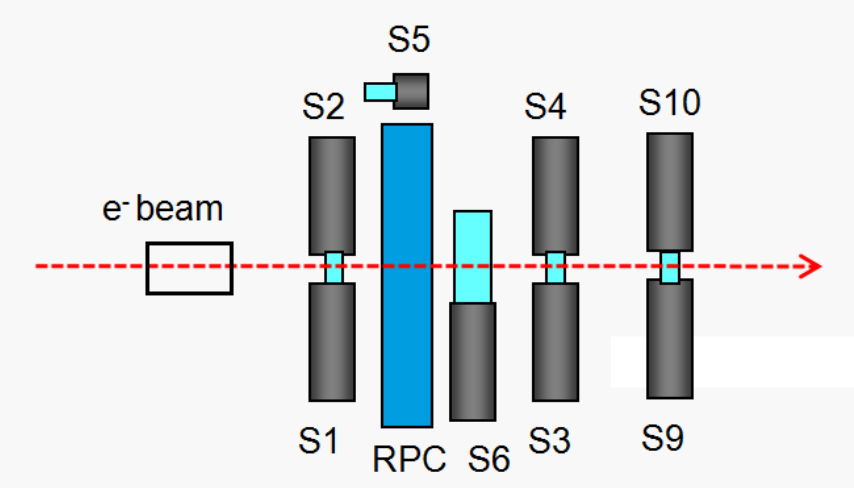

Fig. 4 Schematic view of the test setup. The trigger is determined by $S 1^{\wedge} S 2^{\wedge} S 3^{\wedge} S 4^{\wedge} S 6^{\wedge} \mathrm{RF}$.

By assuming identical multiple scattering in the vertical direction across the strip, the beam profile can be approximately estimated by a two-dimensional gaussian function with a $\sigma \approx$ $2.1 \mathrm{~cm} \times 2.3 \mathrm{~cm}$ :

$$
\phi(x, y)=\frac{\text { Rate }}{2 \pi \sigma_{x} \sigma_{y}} e^{-\left(\frac{x^{2}}{2 \sigma_{x}^{2}}+\frac{y^{2}}{2 \sigma_{y}^{2}}\right)}\left(x_{1} \leq x \leq x_{2}, y_{1} \leq y \leq y_{2}\right)
$$

$\phi(x, y)$ indicates the particle flux and "Rate" indicates the counting rate from the front scintillator (S12). The position distribution of the electrons over the detector area can be expressed by a probability density function:

$$
p(x, y)=\frac{\alpha}{2 \pi \sigma_{x} \sigma_{y}} e^{-\left(\frac{x^{2}}{2 \sigma_{x}^{2}}+\frac{y^{2}}{2 \sigma_{y}^{2}}\right)}\left(x_{1} \leq x \leq x_{2}, y_{1} \leq y \leq y_{2}\right)
$$

$\alpha$ is the normalization factor to make the integration of $p(x, y)$ equal 1 . The average particle flux $\overline{\phi(x, y)}$ can be defined as the expectation value of $\phi(x, y)$ and numerically calculated by a general solution:

$$
\begin{aligned}
& \overline{\phi(x, y)}=\int_{x_{1}}^{x_{2}} \int_{y_{1}}^{y_{2}} \phi(x, y) p(x, y) d x d y \\
& =\int_{x_{2}}^{x_{2}} \int_{y_{1}}^{y_{2}} \frac{\text { Rate }}{2 \pi \sigma_{x} \sigma_{y}} e^{-\left(\frac{x^{2}}{2 \sigma_{x}^{2}}+\frac{y^{2}}{2 \sigma_{y}^{2}}\right)} \cdot \frac{\alpha}{2 \pi \sigma_{x} \sigma_{y}} e^{-\left(\frac{x^{2}}{2 \sigma_{x}^{2}}+\frac{y^{2}}{2 \sigma_{y}^{2}}\right)} d x d y
\end{aligned}
$$

The integration bounds correspond to the size of the area over which the average flux is calculated. In this paper, the bounds are set to the counter size in order to obtain the average flux over the whole counter and the beam profile is determined from the RPC itself. Definition (3) is

\footnotetext{
${ }^{2}$ Unlike the energy loss, that is comparable in both cases.
} 
mathematically sound and very convenient, but it lacks physical insight. A further comprehensive work discussing the difference between local and uniform irradiation is currently under preparation.

\subsection{Summary of results}

The efficiency and time resolution were scanned as a function of the high voltage at low particle flux. The counters show large efficiency plateaux by as much as $600 \mathrm{~V}$, and time resolutions down to $60 \mathrm{ps}$. The high-flux behavior of the two real-size modules is shown in Fig. 5. For comparison, former results under proton beam [4] are also plotted. The 10-gap pad counter was tested under uniform irradiation at GSI in April 2009 (secondary particles from proton reactions @2.5GeV/A). PRPC\#1 and SRPC\#2 were tested under localized irradiation at HZDR in April 2011 (electrons@30MeV). It is evident that the maximum tolerable particle flux reaches up to $25 \mathrm{kHz} / \mathrm{cm}^{2}$ for the tested MRPCs with bulk resistivity of about $10^{10} \Omega \mathrm{cm}$. It can be seen that the counters under an electric field around $110 \mathrm{kV} / \mathrm{cm}$ show better time resolution at high particle fluxes, which indicates that the time resolution of the strip counter can still be improved by optimizing the working voltage. Yet, even for this un-optimized working voltage, the strip counter shows a time resolution around 80ps up to $8 \mathrm{kHz} / \mathrm{cm}^{2}$, enough to cope with the CBM fluxes in regions 2-4.

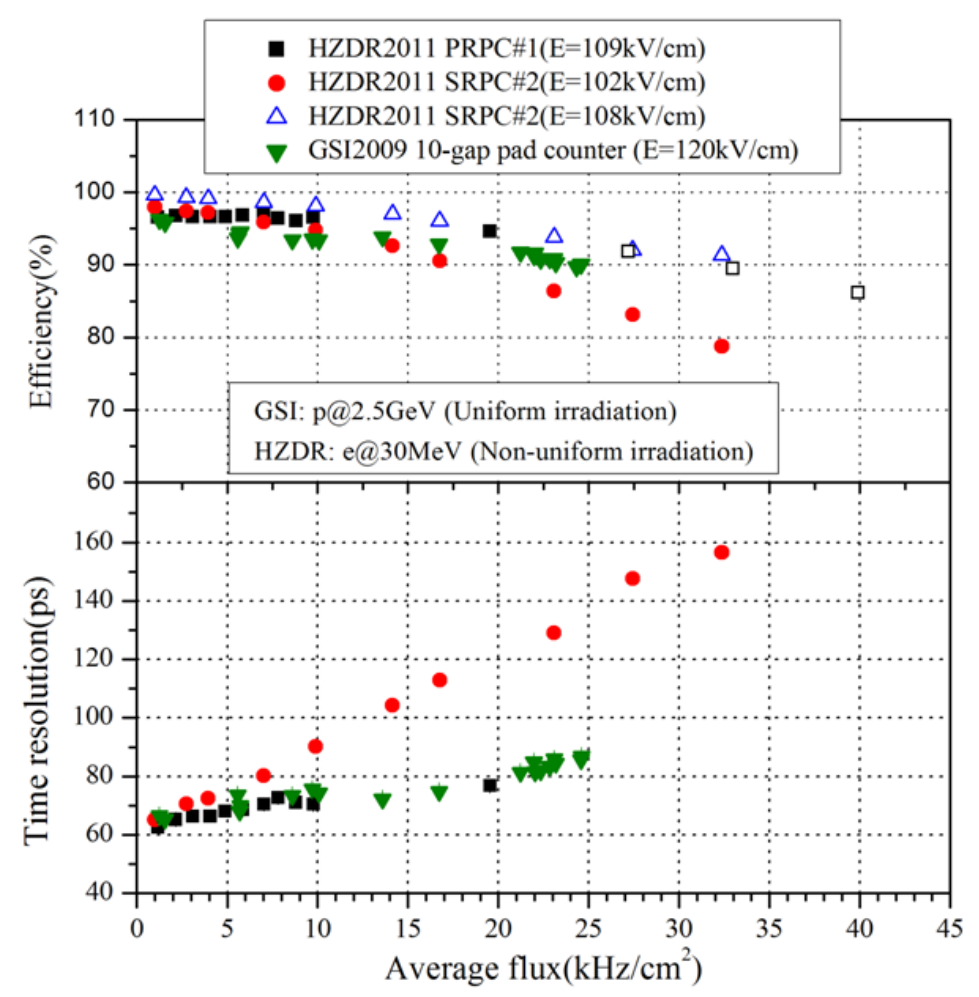

Fig. 5 Measured efficiencies and time resolutions for different runs as a function of particle flux: The 10gap pad counter was tested at GSI in April 2009(secondary particles from proton reactions @2.5GeV/A). PRPC\#1 and SRPC\#2 were tested at HZDR in April 2011 (electrons@30MeV). 


\section{Conclusion}

The rate capability requirement for the most central part of the CBM-TOF wall, up to 20 $\mathrm{kHz} / \mathrm{cm}^{2}$, has been achieved with the newly developed Chinese doped glass. As compared to the current conceptual design of the TOF wall we propose a slight modification to achieve higher realism, by resorting to just one technology that relies on Chinese glass. Based on that, we propose a realistic detector layout for the CBM-TOF wall consisting on five different types of fundamental building modules, with just slight structural differences with respect to modules already tested in beam. The small number of detector families proposed will have an important added value during the production phase.

It is very inspiring that CBM-TOF can be reliably built with detector modules that have been already tested, which gives us additional confidence on the approach. A summary of the performances for all the tested modules is shown in this contribution. Tested with proton and electron beams, the MRPC modules show a maximum rate capability as high as $25 \mathrm{kHz} / \mathrm{cm}^{2}$, thus fulfil the very challenging CBM requirements.

\section{Acknowledgments}

We thank the crew of the ELBE linac for providing excellent beam conditions and warm support during the experiment. We are grateful to the members of the CBM Collaboration for fruitful discussions and their valuable remarks. This work is supported by the National Natural Science Foundation of China under Grant No.s 11020101059, 10775082, 111050110573 and 10979030, It is also supported by Tsinghua University Initiative Scientific Research Program.

\section{References}

[1] FAIR: Facility for Anti-protons and Ion Research, http://www.gsi.de/fair/.

[2] A. Akindinov, et al., Nucl. Instr. and Meth. A 572(2007)676.

[3] V. Ammosov, et al., Nucl. Instr. and Meth. A 576(2007)331.

[4] J. Wang, et al., Nucl. Instr. and Meth. A 621(2010)151.

[5] L.Naumann, et al., Nucl. Instr. and Meth. A 635(2011)113.

[6] M. Petris, et al., Nucl. Instr. and Meth. A 661(2012)151.

[7] I. Deppner, et al., Nucl. Instr. and Meth. A 661(2012)121.

[8] The radiation source ELBE (http://www.fzd.de/pls/rois/Cms?pNid=145\&pOid=10242).

[9] D. Gonzalez-Diaz, 'The ToF wall of the CBM experiment at FAIR', Spring Meeting of the German Physics Society, Giessen, 2007. 